\title{
An ERP study on the executive ability of youths served in plateau.
}

\author{
Xiyuan Wang, Qun Yang*, Wei Gao*, Hui Wang, Junwu Cui, Xiaojun Dong, Bingzhao Wang \\ Fourth Military Medical University, Xi'an, China
}

\begin{abstract}
The study focuses on whether the executive control ability of people is affected by a certain period of stay in plateau. As is known, N2 and LPC (late positive component) time domain reflects people's performance on the conflict detection and response organization. Our study adopts the ERP tech using the Stroop task to examine whether there are differences between people who stay for a certain period in plateau with group in ordinary plain environment. Two groups were tested: the plateau group $(n=14)$ and the plain group $(n=14)$. The electroencephalogram (EEG) continuously recorded at $\mathrm{FCz}$, $\mathrm{Cz}$ and CPz sites with the NeuroLab amplifier. We recorded the signal of the FCZ, CZ and CPZ of N2 and P3 wave amplitude and latency. Compared with the plain environment, both the N2 and LPC elicited by stimuli was prolonged significantly $(p<0.05)$ under the plateau environment than that in the plain, indicating the executive control is impaired in plateau environment.
\end{abstract}

Keywords: Plateau, Cognitive function, Executive control, ERPs, N2, LPC.

Accepted date: February 06, 2018

\section{Introduction}

Recent studies have shown people suffer cognition deterioration after a certain period of stay in plateau [1-4]. Thus, there are plenty of researches in the electrophysiological field using various techniques especially ERP (event-related potential). The deterioration was believed to be caused by hypoxia as largest and most important impact of living at high altitude is hypoxia. Hypoxia is caused by a reduction of oxygen in the air and which affects cognition [5]. It has been welldocumented that the hypobaric-hypoxic environment at high altitude affects the cognition of both permanent residents at high altitude and sojourners [6]. The research on the cognitive impairment of high-altitude hypoxia in people who were born and raised in low-altitude areas and recently serve in Tibet is especially important. Most prior research that has focused on local residents at high altitudes [7,8] or individuals with acute exposure to high altitudes $[9,10]$ has found cognitive impairment caused by high altitude. However, it couldn't conclude the general situation as acute and chronic exposure to high altitudes affects cognition differently [11], and local residents are different from low-altitude residents in terms of genetics and other physiological features [7,12]. The physiological and psychological changes in the immigrant group could reflect the effects of high altitude on cognition better than high-altitude residents or people with acute exposure to high altitudes. Study of cognitive changes in this population can provide a theoretical basis for the provision of cognitive training, prevention of cognitive impairment and other practical applications. Executive control ability consists of a variety of functions like inhibitory control and decision making, which display people's ability to overcome conflicts and to perform actions. Like other kinds of cognition deterioration, executive control ability can be affected after a period of stay in the plateau, which may in some degrees change people's behavior pattern. The Stroop Task can well demonstrate person's executive control ability. Stroop task mainly presents words printed in colored ink and requires the subjects to report the ink color while ignore the intervention of the word meaning $[13,14]$. It consists of 2 parts, namely, the congruent trials and incongruent trials which the word meaning coordinate or uncoordinate with the ink color. The magnitudes of Stroop or interference effects are often used as a proxy of executive control abilities, reflecting the success in monitoring for, detecting, and resolving cognitive conflict. It displays a task of tackling with conflict in order to examine executive ability. Thus, our study aims at using the Stroop task and the ERP technique to explore whether the factors in high altitude environment can affect people's executive ability.

In neurophysiological research, the N2 and LPC component reflects conflict resolving ability which can be seen in the ERP results of a Stroop Task. N2 is mainly distributed in the frontal central area, with a putative source in caudal anterior cingulate cortex (ACC) [15]. It performs as a negative component around $200 \mathrm{~ms} \sim 300 \mathrm{~ms}$. An important function of ACC is detecting conflict during information processing, and alerting higher systems involved in top-down control to resolve conflict. ACC is often activated before reaction during conflict-correcting trials, which is reflected in the amplitudes of $\mathrm{N} 2$ component located in front central area [16]. LPC is the meaning of a late positive-going ERP component, which is found among the parietal scalp sites. It begins around 400-500 ms after the onset of a stimulus and lasts for a few hundred milliseconds.

\section{Subjects}

The experimental group included 14 male youths who born and raised in low-altitude areas now serving in Tibet at an altitude of $3700 \mathrm{~m}$, aged 21-24 years. The control group included 14 male youths live in Weinan at an altitude of $700 \mathrm{~m}$, aged 21-24 years. All subjects signed an informed consent form before the experiment. All the youths were right handed and free of medication for at least $24 \mathrm{~h}$ before testing, with normal or 
Citation: Wang X, Yang Q, Gao W, et al. An ERP study on the executive ability of youths served in plateau. J Psychol Cognition. 2018;3(1): 11-15.

corrected-to-normal vision, without history of head trauma or other medical conditions that could cause cognitive impairment. The study was conducted in accordance with the Declaration of Helsinki and all procedures were carried out with the adequate understanding and written informed consent of the subjects. The study protocol was approved by the Ethics Committee of the Fourth Military of Medical University.

\section{Procedure}

E-prime 2.0 presents stimulus. In the task, word stimuli were the Chinese characters for 'red', 'green', printed in white ink on a black background stadia $100 \mathrm{~cm}$, vertical perspective about $4^{\circ}$. The stimulus is automatically presented. The participants were seated in a reclining chair in a sound attenuated and electrically-shielded room. ERPs were elicited by a Stroop task. They were instructed to press button " $\mathrm{z}$ " to report "red" and press button "m" to report "green". The whole experiment employed 256 stimulus, with the red and green in equal probability (128 times), and the incongruent stimulus 3 times as the congruent stimulus pseudo random. Present time $200 \mathrm{~ms}$, stimulate the average interval (ISI), which is about $2000 \mathrm{~ms}$. Formal experimental participants are asked to do before the experiment task in two groups (12 stimulation/ group) practice to ensure that the participants can properly conduct keystrokes. Participants are asked in the process of experiment binocular-as center screen to reduce eye movement and the blinks of eye as far as possible, and keep their body in a relaxed position. Ocular artifacts were removed from the EEG signal using a regression procedure implemented in the Neuroscan software.

\section{EEG records and analysis}

Using NeuroLab EEG recording system, acquisition of $\mathrm{Cz}, \mathrm{Fcz}$ and $\mathrm{Cpz}$ electrode position of EEG signals was achieved. The electroencephalogram (EEG) was recorded using a system of 41 active electrodes. VEOG and HEOG were recorded with two pairs of electrodes: one pair placed above and below the right eye, and another pair $10 \mathrm{~mm}$ from the lateral canthi. Electrode impedance was maintained below $5 \mathrm{k} \Omega$ throughout the experiment.

The left mastoid was used for recording electrode, with the right side mastoid for recording electrodes, and offline into bilateral mastoid for reference. Grounding forehead, at the same time recorded levels of electric eye and vertical electric eye. A bandpass filter for DC-100 hz, $500 \mathrm{hz}$ sampling frequency, electrode skin contact impedance is less than $5 \mathrm{k} \Omega$. The relevant method to eliminate eye artifact was used for the analysis of the schedule -200-800 milliseconds. After baseline correction, trial of more than $\pm 100 \mu \mathrm{V}$ was eliminated. To eliminate the high frequency noise, ERPs results $30 \mathrm{~Hz}(24 \mathrm{~dB} /$ octave) without phase digital filter were adopted.

Ocular artifacts were removed from the EEG signal using a regression procedure implemented in the Neuroscan software. Epochs were baseline corrected from $2400 \mathrm{~ms}$ to $2200 \mathrm{~ms}$, based on previous. The epoch of interest spanned $200 \mathrm{~ms}$ prior to the response and $800 \mathrm{~ms}$ afterwards. Within this time window the ERP data were digitally filtered (high pass 50.05
Hz, low pass $530 \mathrm{~Hz}, 24 \mathrm{~dB} /$ octave roll off). Artifact rejection was performed to discard epochs contaminated by eye blinks, body movements, and muscle activity. The rejection criterion was a negative or positive change of more than $75 \mathrm{~m} \mathrm{~V}$. Averaged wave forms for each individual subject within each condition were calculated.

\section{Analysis}

Both the two groups produced obvious N2 and LPC. As can be seen from the total average figure, both the congruent and incongruent stimulations of plateau group induced N2 significantly delayed obviously, compared with the plain group (Tables 1-4).

\section{N2}

As effects are generally reported at centro-parietal midline electrodes, analyses were performed at $\mathrm{Cz}$ and $\mathrm{CPz}$. Obvious $\mathrm{N} 2$ component were produced in the two groups. As can be seen from the total average figure, compared with the plain group, the N2 component induced ( $200 \mathrm{~ms} \sim 300 \mathrm{~ms}$ ) of plateau group significantly delayed.

The variance analysis results indicate that the latency of N2 component is significantly different between groups $(\mathrm{F}=5.384$, $\mathrm{p}<0.05)$. N2 component amplitude also differs between groups $(\mathrm{F}=20.685, \mathrm{p}<0.05)$.

\section{LPC}

As can be seen in the Figure 1, the peak of plateau group is significantly higher than that of plain group. In addition, an obvious delay can be observed comparing the incubation period of the two groups. The variance analysis results indicated that the latency of LPC component was significant different between groups $(\mathrm{F}=3.252, \mathrm{p}<0.05)$. LPC component amplitude also differs between groups $(\mathrm{F}=7.863, \mathrm{p}<0.05)$.

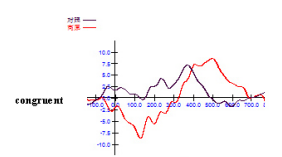

$\mathrm{Cz}$

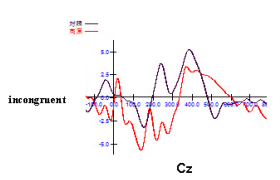

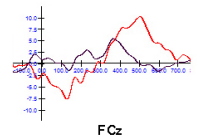

$\mathrm{FCz}$

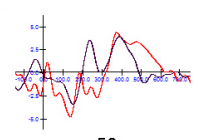

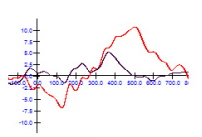

$\mathrm{CPz}$

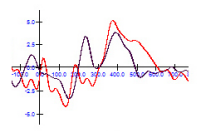

$\mathrm{CP}_{\mathrm{z}}$
Figure 1. LPC, the peak of plateau group is significantly higher than that of plain group.

Table 1. Form Two Groups of participants N2 average amplitude $(u V)$.

\begin{tabular}{lllllll}
\hline Group & \multicolumn{2}{l}{ Incongruent } & \multicolumn{5}{c}{ Congruent } \\
& FCz & $\mathbf{C z}$ & $\mathbf{C P z}$ & $\mathbf{F C z}$ & $\mathbf{C z}$ & $\mathbf{C ~ P z}$ \\
\hline Plateau & -5.37 & -7.59 & -5.73 & -7.94 & -7.70 & -5.32 \\
\hline
\end{tabular}




\begin{tabular}{lllllll}
\hline Plain & -3.72 & -3.05 & -2.61 & -3.99 & -2.32 & -2.63 \\
\hline
\end{tabular}

Table 2. Form Two Groups of participants LPC average amplitude (uV).

\begin{tabular}{lllllll}
\hline Group & \multicolumn{2}{l}{ Incongruent } & \multicolumn{4}{l}{ Congruent } \\
& FCz & Cz & CPz & FCz & Cz & CPz \\
\hline Plateau & 5.94 & 4.97 & 4.16 & 15.41 & 3.31 & 8.78 \\
\hline Plain & 6.25 & 7.24 & 4.82 & 7.34 & 5.27 & 5.93 \\
\hline
\end{tabular}

Table 3. Form Two Groups of participants N2 latency (ms).

\begin{tabular}{lllllll}
\hline Group & \multicolumn{2}{l}{ Incongruent } & \multicolumn{4}{l}{ Congruent } \\
\hline & FCz & Cz & CPz & FCz & Cz & C Pz \\
\hline $\begin{array}{l}\text { Platea } \\
\text { u }\end{array}$ & 272.43 & 261.08 & 265.80 & 253.03 & 247.68 & 257.80 \\
\hline Plain & 252.08 & 252.89 & 259.82 & 220.83 & 215.00 & 222.16 \\
\hline
\end{tabular}

Table 4. Form Two Groups of participants LPC latency (ms).

\begin{tabular}{lllllll}
\hline Group & \multicolumn{2}{l}{ Incongruent } & \multicolumn{4}{l}{ Congruent } \\
\hline & FCz & Cz & CPz & FCz & Cz & C Pz \\
\hline Plateau & 451.40 & 440.29 & 431.13 & 439.70 & 448.29 & 448.81 \\
\hline Plain & 411.86 & 407.74 & 408.35 & 394.11 & 389.56 & 390.09 \\
\hline
\end{tabular}

\section{Discussion}

This study used the Stroop task in order to investigate the influence of plateau environment on people's executive control ability. Using a Stroop task, our study investigated the neural mechanisms responsible for modulation of executive control in healthy young people after long-term exposure to high altitude.

$\mathrm{N} 2$ may reflect the top-down inhibition processing or conflict monitoring processing. As is acknowledged, the N2 component was located in the middle frontal, right inferior frontal, and ACC $[17,18]$ which correlated with conflict monitoring in the response inhibition process [19,20]. Brain imaging findings show that the anterior cingulate cortex (ACC), which is assumed to be activated when conflicts arise, is more activated in both incongruent and congruent than in neutral conditions. This increased ACC activation implies that not only incongruent but also congruent stimuli arouse more conflict than neutral stimuli [21-23]. The N2 became larger in trials that followed low-conflict trials than in trials that followed high-conflict trials [24]. The congruent effect in N2 was also found in studies using classic or numerical Stroop paradigm. As our results showed, the N2 component that plateau group produced have a prolonged latency compared with the plain group in both congruent and incongruent tasks. And the congruent effect is more significant compared with the incongruent task. The amplitude also differs between the plateau group and plain group, which, the average amplitude of $\mathrm{N} 2$ component is higher in plateau group. The prolonged appearance of N2 displays that it is harder for the plateau group to detect the conflict stimuli. On the other hand, the difference in amplitude shows the plateau group may needs to invest more efforts in dealing with certain conflict problems especially the congruent stimuli change. These results may reflect that the ability of detecting and recognizing conflicts gets deteriorated after staying for a certain time in the plateau.

LPC has sometimes referred to as a late component of the P3b [25], which originate from activation in the parietal and temporal regions [26]. Moreover, LPC is related to monitoring whether the stimulus-classification is appropriately translated into action [27]. Thus the LPC could be related to the response organization process. As our results showed, the LPC component that plateau group produced have a prolonged latency compared with the plain group in both congruent and incongruent tasks. This may reflect that the action activation process of the plateau youths can be prolonged as a result disturbing their executive ability.

Previous experimental test have shown that dealing with the problem of response time slowness in the plateau environment may lead to the reduced performance [28,29]. Early studies which used Go/Nogo task found that later NoGo-N2 latency was found in the high-altitude group compared to the lowaltitude group, which suggested prolonged mismatch processing in the high-altitude group [30].Our results confirmed their conclusion in another way using a different task and found the LPC component also differs in the highaltitude and low-altitude group. So what caused these results? According to previous studies Plateau medical studies at home and abroad have shown that the accelerated plateau early brain function change: excited, euphoria, disorientation, and movement coordination, headache, fatigue, etc. Chronic hypoxia prone to causes fatigue, sleepiness, concentration, memory loss and other symptoms. It has been found that, as for migrants living and working in low oxygen conditions, their brain function and sports ability are subjected to different levels of damage, mainly in intelligence. Memory functions especially the instantaneous memory and short-term memory function were significantly impaired. It was also true in the impairment of reaction, judgment, loss of fine operation ability, unsustainable and significantly lower sports ability, quicklylimbs muscle fatigue, and weakness, etc. The youths in plateau environment in the present study adapted to the plateau environment in certain degree. However, research still showed the significant basic cognitive function decrease, and the results of this study could add new evidence in the cognitive research of the plateau and provide a reliable index of electrophysiology in evaluating the effects of plateau environment on the officers and youths capabilities provide.

A long stay in plateau environment can lead to 9 different kinds of psychological problems including: fear of "high", fatigue, irritability, depression, inferiority, pessimistic, anxiety, guilt, and repressed mood. And the chronic hypoxia can affect a series of cognitive functions like memory, sense, attention, etc. According to the results of our research, the participants' prolonged incubation time of N2 and LPC component comes to a conclusion that the certain period of stay in plateau environment may damage people's ability to response to the conflicts and to tackle with the executive tasks which may due 
Citation: Wang X, Yang Q, Gao W, et al. An ERP study on the executive ability of youths served in plateau. J Psychol Cognition. 2018;3(1): 11-15.

to the chronic hypoxia causing lack of oxygen in brain which then disturbs the visual cognitive process. These indicate that people can have problems making decision in the daily shopping or other conflict facing situations after stayed for a certain time in plateau. And it can disturb their lives to somehow. Therefore, how to resolve the problem of executive ability deterioration after the stay at plateau and how long will that be deteriorated still needs further research.

\section{References}

1. Crowley JS, Wesensten N, Kamimori G, et al. Effect of high terrestrial altitude and supplemental oxygen on human performance and mood. Aviat Space Environ Med. 1992;63:696-701.

2. Fowler B, Kelso B. The effects of hypoxia on components of the human event-related potential and relationship to reaction time. Aviat Space Environ Med. 1992;63:510-6.

3. Fowler B, Prlic H. A comparison of visual and auditory reaction time and $\mathrm{P} 300$ latency thresholds to acute hypoxia. Aviat Space Environ Med.1995;66:645-50.

4. Wesensten NJ, Crowley J, Balkin T, et al. Effects of simulated high altitude exposure on long-latency eventrelated brain potentials and performance. Aviat Space Environ Med. 1993;64:30-6.

5. Virués-Ortega J, Garrido E, Javierre C, et al. Human behaviour and development under high-altitude conditions. Dev Sci. 2006;9(4):400-10.

6. Richardson C, Hogan AM, Bucks RS, et al. Neurophysiological evidence for cognitive and brain functional adaptation in adolescents living at high altitude . Clin Neurophysiol. 2011;122(9):1726-34.

7. Wu T, Kayser B. High altitude adaptation in Tibetans. High Alt Med Biol. 2006;7:193-208.

8. Richardson C. Neurophysiological evidence for cognitive and brain functional adaptation in adolescents living at high altitude. Clinl Neurophysiol. 2011;122:1726-34.

9. Hayashi R, Matsuzawa Y, Kubo K, et al. Effects of simulated high altitude on event-related potential (P300) and auditory brain-stem responses. Clin Neurophysiol. 2005;116:1471-6.

10. Takur L, Ray K, Anand J, et al. Event related potential (ERP) P300 after 6 months residence at 4115 meter. Indian J Med Res. 2011;134:113-7.

11. Zubieta-Calleja GR. Human adaptation to high altitude and sea level: Acid-base equilibrium, ventilation and circulation in chronic hypoxia. VDM Verlag. 2010;38-47.

12. Wang B. On the origin of Tibetans and their genetic basis in adapting high-altitude environments. PLoS One. 2011;6:e17002.

13. MacLeod CM. Half a century of research on the Stroop effect: An integrative review. Psychological Bulletin. 1991;109:163-203.

14. Stroop JR. Studies of interference in serial verbal reactions. J Exp Psychol. 1935;18:643-62.
15. VanVeen V, Carter CS. The anterior cingulated as a conflict monitor: FMRI and ERP studies. Physiol Behav. 2002;77:477-82.

16. VanVeen V, Carter CS. The timing of action-monitoring processes in the anterior cingulated cortex. J Cogn Neurosci 2002;14:593-02.

17. Bokura H, Yamaguchi S, Kobayashi S. Electrophysiological correlates for response inhibition in a Go/NoGo task. Clin Neurophysiol. 2001;112:2224-32.

18. Karch S. Separating distinct aspects of the voluntary selection between response alternatives: N2-and P3-related BOLD responses. Neuroimage. 2010;51:356-64.

19. Nieuwenhuis S, Yeung N, van den Wildenberg W, et al. Electrophysiological correlates of anterior cingulate function in a go/no-go task: Effects of response conflict and trial type frequency. Cogn Afect Behav Neurosci. 2003;3:17-26.

20. Botvinick MM, Cohen JD, Carter CS. Conflict monitoring and anterior cingulate cortex: An update. Trends Cogn Sci. 2004;8:539-46.

21. Bench CJ, Frith CD, Grasby PM, et al. Investigations of the functional anatomy of attention using the Stroop test. Neuropsychologia. 1993;32:907-22.

22. Bush G, Luu P, Posner MI. Cognitive and emotional influences in anterior cingulate cortex. Tren Cognitive Sci. 2000;4:215-22.

23. Carter CS, Mintun M, Cohen JD. Interference and facilitation effects during selective attention: An $\mathrm{H} 215 \mathrm{O}$ PET study of Stroop task performance. NeuroImage. 1995;2:264-72.

24. Wijers AA, Mulder G, Okita T, et al. Attention to color: An analysis of selection, controlled search and motor activation, using event-related potentials. Psychophysiology. 1989;26:89-109.

25. Finnigan S, Humphreys MS, Dennis S, et al. ERP‘old/ new' effects: Memory streng than d decisional factor (s). Neuropsychologia. 2002;40:2288-304.

26. Polich J. Updating P300: An integrative theory of P3a and P3b. Clin. Neurophysiol. 2007;118:2128-48.

27. Verleger R, Ja'skowski, Wascher E. Evidence for an integrative role of $\mathrm{P} 3 \mathrm{~b}$ in linking reaction to perception. $\mathrm{J}$ Psychophysiol. 2005;19:165-81.

28. Schmitt BM, Munte TF, Kutas M. Electrophysiological estimates of the time course of semantic and phonological encoding during implicit picture naming. Psychophysiology. 2000;37:473-84.

29. Gajewski PD, Stoerig P, Falkenstein M. ERP-correlates of response selection in a response conflict paradigm. Brain Res. 2008;1189:127-34.

30. Ma H. Long-term exposure to high altitude affects response inhibition in the conflict-monitoring stage. Sci Rep. 2015;5:13701.

\section{*Correspondence to:}

Qun Yang

Fourth Military Medical University 
China,

Tel: 8613709241788
E-mail: yangqun@fmmu.edu.cn 